\title{
AGN in Submm Galaxies - Combining the Power of Chandra and ALMA
}

\author{
Sharon Xuesong Wang ${ }^{1}$ \\ The Pennsylvania State University \\ State College, Pennsylvania, USA \\ E-mail: xxw131eastro.psu.edu

\section{The LESS/ALESS Collaboration} \\ University of Durham, MPIA, MPIfR, etc. \\ UK, Germany, etc.
}

Submillimeter Galaxies (SMGs) are massive, distant galaxies that are observed to have extremely high star formation rates, and they are believed to be possibly the progenitors of massive elliptical galaxies. Previous studies have found that they have a higher likelihood to harbour Active Galactic Nuclei (AGNs), likely in the pre-quasar phase, but these studies were controversial due to the inhomogeneity and poor angular resolution of earlier submillimeter surveys. Our work sets out to put more stringent constraints on the links between AGN activity in SMGs by combining the powers of ALMA and Chandra observations in the Extended Chandra Deep Field South (E-CDF-S). Our SMG samples are from the ALMA LABOCA ECDFS-S Submillimeter Survey (ALESS), which is the first fully identified, most homogeneous, and the largest contiguous survey at $870 \mu \mathrm{m}$. We have found $10 \mathrm{X}$-ray sources associated with SMGs, all at $z>1.5$, and 8 of them were identified to be AGNs. Five of them have enough Xray counts to enable a proper spectral fit. One of these five is a previously known quasar, and appears to be unabsorbed. Three of the remaining four appear to be heavily absorbed $\left(\mathrm{N}_{\mathrm{H}}>10^{23}\right.$ $\mathrm{cm}^{-2}$ ), among which two sources have intrinsic X-ray luminosity larger than $10^{44} \mathrm{erg} / \mathrm{s}$ and can be classified as obscured quasars. The last one is moderately obscured. The remaining five of the ten that do not have enough X-ray counts for spectral fit also exhibit different levels of absorption. An analysis on AGN fraction, taking into account the spatial variation of survey sensitivity, yields an $\mathrm{f}_{\mathrm{AGN}}$ of $17 \%(+16 \%-6 \%)$ for SMGs hosting AGN with intrinsic luminosity larger than $\sim 10^{43} \mathrm{erg} / \mathrm{s}$, which is consistent within error bars with previous analyses. The AGN fraction of this group of SMGs in the E-CDF-S appears to be consistent with the $\mathrm{f}_{\mathrm{AGN}}$ values for galaxies at similar redshifts with comparable masses and star formation rates. The results presented here are preliminary.

Nuclei of Seyfert galaxies and QSOs - Central engine \& conditions of star formation November 6-8, 2012

Max-Planck-Insitut für Radioastronomie (MPIfR), Bonn, Germany

1 Speaker 


\section{Introduction}

Submillimeter galaxies (SMGs) are massive, distant galaxies that are very luminous in the observed-frame submillimeter (submm) band. They are known as the "distant" cousins of the local ultra-luminous infrared galaxies (ULIRGs) as they share common properties but differ from each other even more. Like ULIRGs, SMGs are highly star forming, but with even larger star formation rate (SFR) of typically $\sim 1000 \mathrm{M}_{\odot}$ /year (Blain et al. 2002; Smail et al. 2002). They are more massive galaxies than ULIRGs, with stellar mass about $10^{11} \mathrm{M}_{\odot}$ though they are typically at redshift $\mathrm{z} \sim 2$, only $\sim 3.3 \mathrm{Gyr}$ after the Big Bang. They are also thought to have different star formation patterns from the ULIRGs: while ULIRGs are known to have concentrated star formation at their cores, SMGs seem to have star formation at larger scale over the galaxies (Coppin et al. 2012).

They are also thought to be related to the quasar activities near redshift $\mathrm{z} \sim 2$, and are thought to have a large AGN duty cycle and to host pre-quasar black holes (Alexander et al. $2005 \mathrm{a}, \mathrm{b})$. This is perhaps not surprising, since there are known correlation between the occurrence rate of Active Galactic Nuclei (AGNs) and the stellar mass of galaxies (e.g. Xue et al. 2010, Aird et al. 2011). These massive galaxies are also thought to be the progenitors of the local massive elliptical galaxies (Chapman et al. 2005), and if true, this is consistent with the picture of merger-driven galaxy growth and the co-evolution of the central black hole (e.g., Hopkins et al. 2008).

However, the results from previous studies on AGNs in SMGs are controversial, and do not agree on the AGN fraction and duty cycle of the SMGs (Alexander et al. 2005a; Laird et al. 2010). This is mainly due to the extremely challenging nature of unambiguously identifying the counterparts of SMGs, because the last generation submm surveys had poor angular resolution (Chapman et al. 2005; Weiss et al. 2009; Biggs et al. 2011). In addition, previous works were done in the Chandra Deep Field North (CDF-N) region, where multiple submm surveys were conducted over different sub-regions of CDF-N, and the combined submm sensitivity map is inhomogeneous over the region.

The LABOCA Extended Chandra Deep Field South (E-CDF-S) Submm Survey (LESS; Weiss et al. 2009) is the first contiguous and uniform submm survey, and it covers the most Xray sensitive region on the sky observed with Chandra, the 4 Ms CDF-S survey (Xue et al. 2011; X11). There are 126 bright submm sources found in the LESS survey, and they are followed up by the Atacama Large Millimeter/submillimeter Array (ALMA) in Cycle 0 in the ALMA LABOCA E-CDF-S Submm Survey (ALESS; Hodge et al. 2013). The exquisite sensitivity and angular resolution of ALMA $(<0.5$ ") provides us the opportunity to unambiguously identify the X-ray counterparts of SMGs in this field for the first time, and to study the AGNs at the center of these highly star forming galaxies. 


\section{AGN Identification}

\subsection{Matching the X-ray Sources and SMGs}

We first aim to find secure X-ray counterparts to the ALESS SMGs. The ALESS main source catalog contains 99 sources in total (Hodge et al. 2013), with 91 sources lying within the E-CDF-S region. Our X-ray catalog consists of 892 sources from the $4 \mathrm{Ms}$ CDF-S (X11) and 762 sources from the 250 ks E-CDF-S (Lehmer et al. 2005; L05). The X-ray catalog includes the main and supplementary catalog sources from L05 and X11, as well as additional sources from a WAVDETECT catalog with a false-positive probability threshold of $<10^{-5}$, which is larger than used for selecting main or supplementary sources in L05 and X11, but if matched to submm sources, these sources are likely to be robust.

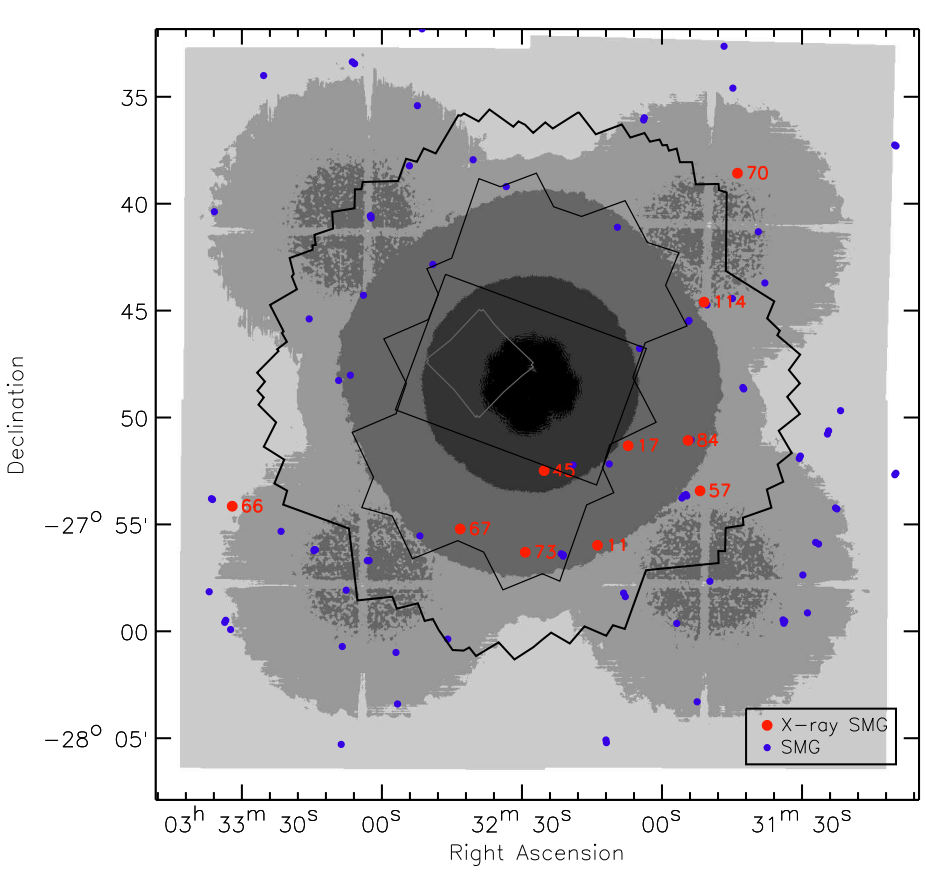

Figure 1: X-ray sensitivity map for the E-CDF-S region. The grayscale levels, from black to light gray, represent areas with flux limits of $<4 \times 10^{-17}, 4 \times 10^{-17}$ to $10^{-16}, 10^{-16}$ to $3.3 \times 10^{-16}, 3.3 \times 10^{-16}$ to $10^{-15}$, and $>10^{-15} \mathrm{erg} / \mathrm{cm}^{2} / \mathrm{s}$, respectively. The large red dots mark the X-ray detected SMGs, while the blue dots are other SMGs in the ALESS main catalog. X-ray detected SMGs are labeled with their short LESS IDs. The solid lines show the regions for, from outer to inner, the 4 Ms CDF-S, GOODS-S, CANDELS GOODS-S Deep Field, and Hubble Ultra Deep Field.

We employed likelihood-ratio matching method detailed in Luo et al. (2010) to search for X-ray counterparts for the SMGs. We found 10 matches with an estimated false match probability of $\sim 3 \%$ (expected number of false match $=0.3$ ). The X-ray detected SMGs are shown in the X-ray sensitivity map in Figure 1.

\subsection{AGN or Starburst Classification}

We then proceed to analyze if the X-ray emission detected in these 10 SMGs is from AGN or can be fully explained by just star formation in these starburst galaxies. Left panel of Figure 2 shows an example of our classification method through comparison of rest-frame X-ray luminosity, $\mathrm{L}_{0.5-8 \mathrm{keV}}$, (not absorption corrected) and radio luminosity in the $1.4 \mathrm{GHz}$ band, converted from observed $1.4 \mathrm{GHz}$ flux assuming a radio spectral index of $\alpha=0.8$. Any SMGs with X-ray luminosity that is a factor of 5 larger than the amount expected from pure star formation, as estimated by the radio luminosity, are classified as AGN hosts. As shown in the left panel of Figure 2, source No. 17, 45 and 67 (their short LESS IDs) are not classified as 
AGN hosts using this method, while all other 7 SMGs are. However, LESS 17 has a very hard $\mathrm{X}$-ray spectrum, and is therefore classified as AGN under other criteria we used.

Other criteria that we used to see if an SMG hosts AGN, including: (1) having an intrinsic, absorption-corrected X-ray luminosity of larger than $3 \times 10^{42} \mathrm{erg} / \mathrm{s}$; (2) having an X-ray spectral index $\Gamma<1.0$; (3) X-ray luminosity being a factor of 5 or larger as expected from star formation, which is estimated by stellar mass and infrared luminosity following Lehmer et al. (2010); (4) observed infrared flux in the IRAC $3.6 \mu \mathrm{m}$ band is smaller than $1 / 10$ of the observed X-ray flux in $0.5-8 \mathrm{keV}$. Combining all these criteria together, except for LESS 45 and 67 , which have relatively low X-ray luminosity and soft spectral indices that are consistent with X-ray coming from star formation, the other 8 out of 10 SMGs are classified as AGN hosts.

\subsection{Spectral Properties}

Among the 8 AGNs, one is a known quasar (LESS 66), and it appears to be unabsorbed. The other 7 AGNs all show moderate to high obscuration, including the heavily obscured AGN at redshift of 4.76 (LESS 73) reported by Gilli et al. (2011).

The right panel of Figure 2 is a schematic view of how obscured these AGNs appear to be. Seven AGNs appear to have $\mathrm{N}_{\mathrm{H}}$ around $10^{23} \mathrm{~cm}^{-2}$ or even higher. Five out of ten SMGs have enough X-ray photon counts $(>100)$ to enable a proper spectral fitting, and the results shown in Figure 2 are consistent with our spectral fits.
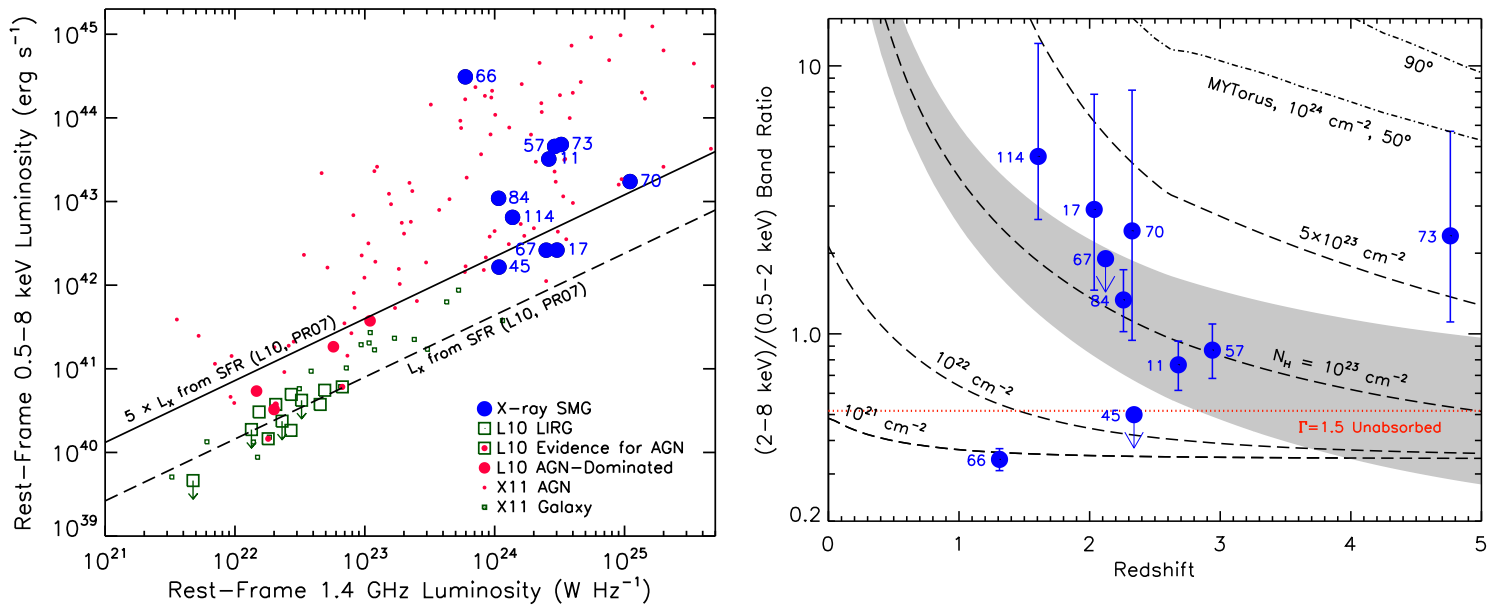

Figure 2: Left: An example of our AGN identification method: the rest-frame $0.5-8 \mathrm{keV}$ luminosity (no absorption correction) vs. the rest-frame $1.4 \mathrm{GHz}$ monochromatic luminosity. The X-ray detected SMGs are big blue dots labeled with their short LESS IDs. The dashed line marks the $\mathrm{L}_{0.5-8 \mathrm{keV}}$ to $\mathrm{L}_{1.4 \mathrm{GHz}}$ ratio as calculated by converting $\mathrm{L}_{1.4 \mathrm{GHz}}$ into star formation rate (SFR) following Persic \& Rephaeli (2007; PR07) and references therein, and then converting SFR into $\mathrm{L}_{0.5-8 \mathrm{kev}}$ (with no dependence on the galaxy stellar mass $\mathrm{M}_{\star}$ ) following Lehmer et al. (2010; L10). The solid line shows where $\mathrm{L}_{0.5-8 \mathrm{keV}}$ is a factor of 5 larger than predicted from $\mathrm{L}_{1.4 \mathrm{GHz}}$ and SFR using PR07 and L10 correlations. Sources above the solid line are classified as AGNs (similar to the criterion adopted in Alexander et al. 2005b and X11). Right: The band ratio between the $2-8 \mathrm{keV}$ (hard) and the $0.5-2 \mathrm{keV}$ (soft) bands (i.e., X-ray hardness) vs. redshift for our SMGs. Plotted are $1 \sigma$ error bars for the band ratios. Note that for ALESS 45.1 and ALESS 67.1, the net counts are low and the backgrounds are high, and thus the band ratios are not well constrained and $90 \%$ upper limits are provided here. The dashed lines are tracks for AGN spectral models described by a power-law with the effects of both Galactic and intrinsic absorption with the intrinsic photon index $\Gamma_{\text {int }}$ fixed to 1.8 (XSPEC wabs*zwabs*zpow). The shaded region is for models with a varying $\Gamma_{\text {int }}=1.8 \pm 0.5$ for $\mathrm{N}_{\mathrm{H}}=10^{23} \mathrm{~cm}^{-2}$. The dash dotted lines are tracks calculated using the MYTorus model (Murphy \& Yaqoob 2009), with $\Gamma=1.8, \mathrm{~N}_{\mathrm{H}}=10^{24} \mathrm{~cm}^{-2}$, and inclination angles of 50 and 90 degrees. The red dotted line manks the expected hardness ratio for $\Gamma=1.5$ and no intrinsic absorption, which could also describe a typical starbust/HMXB population (e.g., Teng et al. 2005; Lehmer et al. 2008). Most of the X-ray detected SMGs appear to have high levels of obscuration. 


\section{AGN Fraction among Submm Galaxies}

We then estimate the AGN fraction in these SMGs. We follow the method discussed in Lehmer et al. (2007) and Silverman et al. (2008) to calculate the cumulative AGN fraction $f_{A G N}$ for SMGs hosting AGNs with a certain intrinsic (absorption corrected) $0.5-8 \mathrm{keV} \mathrm{X-ray}$ luminosity value or higher. This method takes into account the spatial inhomogeneity in X-ray sensitivity limit and compensates for the bias induced by such X-ray catalogs when estimating AGN fraction. It computes the contribution from each AGN to the cumulative AGN fraction, and since we have only 8 AGNs in our sample, the X-ray luminosity values we chose to compute $f_{A G N}$ at are simply the X-ray luminosities of these 8 AGNs. The results are plotted in Figure 3. About $17 \%$ of SMGs host AGNs with intrinsic Xray luminosity of $7.8 \times 10^{42} \mathrm{erg} / \mathrm{s}$ or larger. This is roughly consistent with previous results, though previous studies are relatively more uncertain due to non-uniform

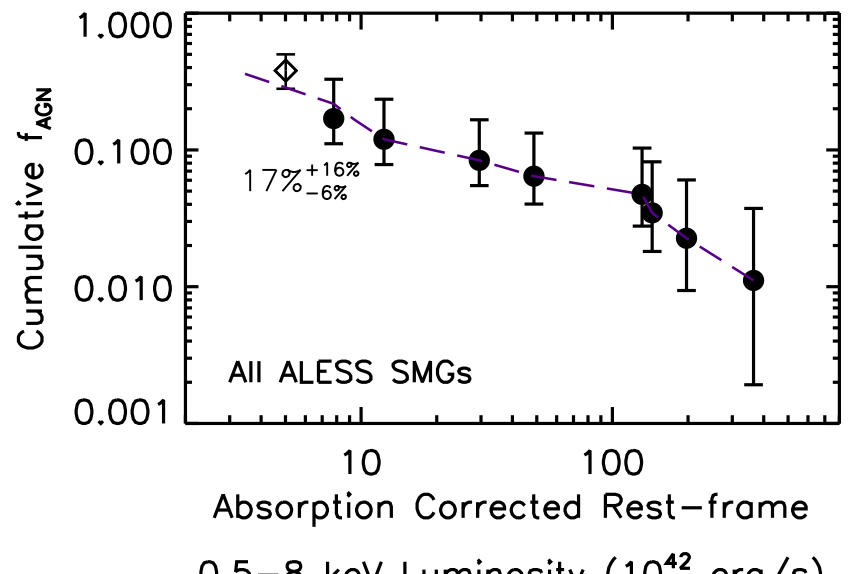$$
0.5-8 \mathrm{keV} \text { Luminosity }\left(10^{42} \mathrm{erg} / \mathrm{s}\right)
$$

Figure 3: Cumulative $A G N$ fraction $f_{A G N}$ as a function of $0.5-8$ $\mathrm{keV}$ intrinsic (rest-frame absorption-corrected) luminosity. The calculation of AGN fraction is done following Lehmer et al. (2007) and Silverman et al. (2008). Each point represents the AGN fraction $f_{A G N}$ in the SMG sample for AGNs with a certain value of intrinsic $0.5-8 \mathrm{keV}$ luminosity or larger. The diamond is $\mathrm{f}_{\mathrm{AGN}}$ estimated in Alexander et al. (2005b). submm survey and less robust counterpart identification.

While $17 \%$ AGN fraction is relatively high compared to all field galaxies at redshift $z=1-3$ or so, this value is actually not surprising given the high stellar mass and high star formation rate of SMGs. Indeed, if compared to galaxies with similar stellar mass $\left(\sim 10^{11} \mathrm{M}_{\odot}\right)$ or galaxies with similar star formation rate $\left(\sim 1000 \mathrm{M}_{\odot}\right.$ /year), they have comparable AGN fraction (see, e.g. Xue et al. 2010). However, it is yet to be understood why massive or highly star forming galaxies have higher AGN fraction.

Among the $10 \mathrm{X}$-ray detected SMGs in our sample, 8 are AGN-dominated in the X-ray. It is reasonable to speculate that if we go deeper in X-ray, more AGNs in SMGs will be revealed, especially considering the high obscuration we saw in our AGN sample. With the exquisite sensitivity and angular resolution of ALMA, we are just beginning to understand the nature of SMGs and the AGNs they host. Are they truly merger driven? What is their difference from the local ULIRGs? Are the AGNs we found in SMGs going to grow into the powerful quasars we see around redshift $\mathrm{z} \sim 2$ ? As more samples of SMGs accumulate and more secure multiwavelength counterparts identified, we are getting closer and closer to answering these questions and to revealing the role that AGNs may have played in the course of galaxy evolution and cosmic star formation, which was one of the key questions of this conference. 


\section{References}

[1] Aird, James, Coil, Alison L., Moustakas, John, et al. ApJ 746 (2012) 90

[2] Alexander, D. M., Bauer, F. E., Chapman, S. C., et al. ApJ 632 (2005) 736

[3] Alexander, D. M., Smail, I., Bauer, F. E., et al. Nature 434 (2005) 738

[4] Biggs, A. D., Ivison, R. J., Ibar, E., and et al. MNRAS 413 (2011) 2314

[5] Blain, Andrew W., Smail, Ian, Ivison, R. J., et al. PhR 369 (2002) 111

[6] Chapman, S. C., Blain, A. W., Smail, Ian, et al. ApJ 622 (2005) 772

[7] Coppin, K. E. K., Danielson, A. L. R., Geach, J. E., et al. MNRAS 427 (2012) 520

[8] Gilli, R., Su, J., Norman, C., et al. ApJ 730 (2011) L28

[9] Hodge et al. ApJ submitted (2013)

[10] Hopkins, Philip F., Hernquist, Lars, Cox, Thomas J., et al. ApJS 175 (2008) 356

[11] Laird, Elise S., Nandra, Kirpal, Pope, Alexandra, et al. MNRAS 401 (2010) 2763

[12] Lehmer, B. D., Alexander, D. M., Bauer, F. E., et al. ApJ 724 (2010) 559

[13] Lehmer, B. D., Brandt, W. N., Alexander, D. M., et al. ApJS 161 (2005) 21

[14] Lehmer, B. D., Brandt, W. N., Alexander, D. M., et al. ApJ 681 (2008) 1163

[15] Lehmer, B. D., Brandt, W. N., Alexander, D. M., et al. ApJ 657 (2007) 681

[16] Luo, B., Brandt, W. N., Xue, Y. Q et al. ApJS 187 (2010) 560

[17] Murphy, Kendrah D. and Yaqoob, Tahir. MNRAS 397 (2009) 1549

[18] Persic, M. and Rephaeli, Y.. A\&A 463 (2007) 481

[19] Silverman, J. D., Mainieri, V., Lehmer, B. D., et al. ApJ 675 (2008) 1025

[20] Smail, Ian, Ivison, R. J., Blain, A. W., et al. MNRAS 331 (2002) 495

[21] Teng, Stacy H., Wilson, A. S., Veilleux, S., et al. ApJ 633 (2005) 664

[22] Weiss, A., Kov\&aacute;cs, A., Coppin, K., et al. ApJ 707 (2009) 1201

[23] Xue, Y. Q., Brandt, W. N., Luo, B., et al. ApJ 720 (2010) 368

[24] Xue, Y. Q., Luo, B., Brandt, W. N., et al. ApJS 195 (2011) 10 